\title{
Using network centrality measures to manage landscape
}

\section{connectivity.}

\author{
Ernesto Estrada $^{1^{*}}$ and Örjan Bodin ${ }^{2,3}$ \\ ${ }^{1}$ Complex Systems Research Group, RIAIDT, Edificio CACTUS, University of Santiago \\ de Compostela, Santiago de Compostela 15782, Spain and ${ }^{2}$ Stockholm Resilience Centre, \\ Stockholm University, 10691 Stockholm, Sweden and ${ }^{3}$ Department of Government, \\ Uppsala University, 75105 Uppsala, Sweden
}

* Corresponding author. E-mail: estrada66@yahoo.com. Fax: +34 981547077 


\begin{abstract}
We use a graph-theoretical landscape modeling approach to investigate how to identify central patches in the landscape as well as how these central patches influence (1) organism movement within the local neighborhood, and (2) the dispersal of organisms beyond the local neighborhood. Organism movements were theoretically estimated based on the spatial configuration of the habitat patches in the studied landscape. We find that centrality depends on the way the graph-theoretical model of habitat patches is constructed, although even the simplest network representation, not taking strength and directionality of potential organisms flows into account, still provides a coarse-grained assessment of the most important patches according to their contribution to landscape connectivity. Moreover, we identify (at least) two general classes of centrality. One accounts for the local flow of organisms in the neighborhood of a patch and the other for the ability to maintain connectivity beyond the scale of the local neighborhood. Finally, we study how habitat patches with high scores on different network centrality measures are distributed in a fragmented agricultural landscape in Madagascar. Results show that patches with high degree-, and betweenness centrality are widely spread, while patches with high subgraph- and closeness centrality are clumped together in dense clusters. This finding may enable multi-species analyses of single-species network models.
\end{abstract}

Key words: landscape connectivity, complex networks; graph theory; conservation; network centrality; habitat patch; landscape ecology; factor analysis; landscape management, subgraph centrality; betweenness centrality; network representations 


\section{INTRODUCTION}

Habitat loss, as a consequence of increased human land use and expropriations of natural habitats, is increasingly being considered as one of the major threats to biodiversity and functional ecosystems (Meffe et al. 2002, Fahrig 2003). Habitat loss basically gives rise to two different, although closely related, spatial consequences. First, the amount of natural habitat obviously decreases as more land is converted for expample agricultural production. Secondly, the conversion of natural habitat decreases the connectivity of the landscape, i.e.,, it increases the level of habitat fragmentation. The focus of this paper is on this latter consequence of habitat loss. Connectivity is, on a general level, the degree to which the spatial pattern of scattered habitat patches in the landscape facilitates or impedes the movement of organisms (Taylor et al. 1993). The persistence of spatially-structured species populations - metapopulations - is strongly related to the connectivity of the landscape (Hanski and Ovaskainen 2003). If the connectivity of the landscape is too low, subpopulations get isolated, and for instance the possibility of recoveries following local extinctions decreases since successful recolonization is dependent on the dispersal of species throughout the landscape (Hanski and Ovaskainen 2003, Hanski 1994, Bascompte and Solé 1996). On the other hand, even if habitat fragmentation has decreased the number of large coherent patches of habitat in the landscape, a sufficiently high level of connectivity may still provide for sufficiently large areas of reachable habitat, as seen by species capable of moving from patch to patch (Lundberg and Moberg 2003). Thus, management and planning should take these, and other, aspects of landscape connectivity into account in order to provide ecologically functional and resilient landscapes in for instance, designing natural reserves and in green 
area planning of urban and/or semi-urban areas (Bengtsson et al. 2003, Lee and Thompson 2005, Bodin and Norberg 2007). Within this problem domain of management, one of the challenges is to differentiate the impact individual habitat patches have on the overall connectivity of the fragmented landscape. Often, not all natural habitats can be preserved since a multitude of different societal interests in land usages has to be balanced in a typical management situation (Bengtsson et al. 2003). The problem that not all habitat patches can be preserved begs the question; Which ones shall managers choose to conserve? Furthermore, the flip-side of that question is; Which patches can be exploited while still minimizing the negative ecological consequences?

At present there is an abundance of metrics that quantify landscape pattern (Gustafson 1998), but how well these metrics explain ecological processes is still largely unknown (Hargis et al. 1998, Tischendorf 2001). Ecological interpretability is however of crucial importance for any spatial configuration metric in order to make it useful in management ( $\mathrm{Li}$ and $\mathrm{Wu} 2004)$. One promising approach fulfilling this criterion is the graph-theoretical perspective on landscape connectivity (Keitt et al. 1997, Urban and Keitt 2001, Pascual-Hortal and Saura 2006, Bodin and Norberg 2007, Campbell Grant et al. 2007, Minor and Urban 2007, Calabrese and Fagan 2004). This approach merges population processes, such as dispersal, with spatial patterns of habitat patches - on the level of landscapes - in order to attain process-based measures of connectivity (Urban and Keitt 2001). The basic idea is to depict landscapes of fragmented habitat patches as networks where the patches are the nodes and the links are the possible dispersal pathways for dispersing species. 
For this study, our focus is to elaborate on different methods, using the graphtheoretical modeling approach to landscape connectivity, to assess and differentiate the importance of individual habitat patches in relation to their impact on different aspects of the landscape's connectivity (Pascual-Hortal and Saura 2006). Within the broad interdisciplinary field of network analysis, the concept of centrality is used to assess the individual capability of nodes to influence others (Wasserman and Faust 1994, Estrada 2007a). In short, the concept of centrality is manifested through a family of networkcentric metrics designed to assess, broadly defined, individual nodes' level of influence based on their structural position relative to others in the network. Our detailed objective with this study is to examine the applicability of a range of such centrality measures in studying individual habitat patches contribution to the landscape connectivity. The goal is to develop methods helpful in real world situations where management needs to make informed decisions on which habitat patches to conserve, and which ones to exploit. The current work also connects to the evolving field of network-oriented studies of different complex systems ranging from social and technological to biological and ecological (Strogatz, 1998; Albert and Barabási, 2002; Newman, 2003; Boccaletti et al. 2006).

Here we first briefly present a heavily fragmented agricultural landscape in southern Madagascar (Bodin et al. 2006) which is thereafter used throughout the paper for the different analyses. We proceed with presenting some different approaches in constructing network representations of highly fragmented landscapes, followed by a presentation of a selected set of centrality measures. Then, by applying these different network representations on the Madagascar landscape, we examine (1) how the selected set of centrality measures behave in relation to these different kinds of network representations, 
and (2) how the centrality measures differ among each other. We show that these measures, on a general level, can be reduced to two different aspects of centrality, both of potentially great importance in assessing individual patches' contributions to landscapes connectivity. These aspects are suggested to encompass (1) a patch's contribution to the magnitude of species interpatch movements on a local geographical level, and (2) the criticality of an individual patch when it comes to providing large-scale connectivity, i.e.,, the importance of the individual patch in preventing the network of habitat patches to divide into smaller and isolated compartments, or islands, of patches. Finally we present an analysis of the geographical distribution of the patches with high scores on different measures of centrality, and suggest a multi-species analysis using speciesspecific network representations of fragmented landscapes.

\section{METHODS}

\section{Study area - southern Madagascar}

The study area used throughout the analyses in this paper is located in the Androy region in the very south of Madagascar, and it is described in details elsewhere (Bodin et al. 2006; Bodin and Norberg 2007). It consists of hundreds of small forest patches ( $<1$ to 95 ha) that are scattered in an agricultural landscape (Fig. 1). This study area was chosen since it provides an illustrative example of a landscape that has been fragmented as a result of agriculture. In spite of its limited size, the forest patches provide habitat for our target species; the Ring-tailed Lemur (Lemur catta). L. catta is an important seed disperser in the area since it forages on fruits of many different plant species in the area (see Bodin et al. 2006 and references therein). Hence their movement between different forest patches, and in the matrix, can potentially disperse seeds throughout the landscape 
and accordingly it may contribute significantly to this important ecosystem service (Millennium Ecosystem Assessment 2003). A spatial analysis of the fragmented forest patches, as experienced by L. catta, is therefore of interest in order to gain insights in how well the landscape's spatial configuration supports seed dispersal. In this study, we build on a previous graph-theoretical analysis of how L. catta may experience the studied landscape; thus details on assessments of movement capability and habitat suitability are not included here but can be found in Bodin et. al. (2006).

Insert Fig. 1 about here.

\section{Graph representations of fragmented landscape}

The basic modeling approach in this paper is to present a landscape of scattered habitat patches as a network consisting of nodes and links (Keith et al. 1997). Each habitat patch is here represented as a node, and a link between any two nodes represents connectivity between the two corresponding patches. If two patches are connected, the target species is able to move between these patches thus implying there is a potential flow of organisms between the two. There are a number of different methods available to estimate the level of connectivity between any two patches (Keitt et al. 1997, Bunn et al. 2000, Verbeylen 2003, Bodin et al. 2006). These methods represent different ways of quantifying the effective distance, as experienced by the target species, between the patches in question. In its simplest form, the effective distance will be the same as the geographical distance. In heterogeneous landscapes, the effective distance between any two patches should be assessed based on the permeability of the specific land types separating these. In any case, longer effective distance means less connectivity (and flow of organisms) between the patches. In this study, we estimated the flow of organisms between any two patches by applying the underlying assumptions behind the Incident 
Function Model (Hanski 1994). Thus, the flow was assumed to decrease exponentially with increasing inter-patch distance and to increase proportionally to the square root of the habitat patch area (see further details in Bodin et al. 2006).

After assessing the connectivity between all pairs of patches in the landscape, the resulting network will represent all possible movement paths throughout the landscape, i.e.,, it will represent the landscape's structure of connectivity as experienced by the target species.

In general, a network of fragmented patches is represented as a $N x N$ matrix, called an adjacency matrix $\mathbf{A}$ where each patch (of a total of $N$ patches) is represented by one row and one column (Harary 1969). Then, each element $A_{i j}$ represents the level of connectivity between patch $i$ and patch $j$. The most general representation of a landscape network is a so-called weighted directed graph, where each element in $\mathbf{A}$ is weighted and $A_{i j}$ is not necessarily the same as $A_{j i}$ (Harary 1969). This representation is often simplified by dichotomizing all elements based on a threshold weight, and the adjacency matrix can also be made undirected ( $A_{i j}$ equals $A_{j i}$ ). These different representations are described below. i) Weighted-directed network. In this representation the weight of the network links are proportional to an estimated flow of organisms potentially moving from one patch $i$ to another patch $j$ as explained earlier. Obviously, the number of organisms flowing from $i$ to $j$ are not necessarily the same as those flowing in the reverse direction. Thus, the network is directed and its adjacency matrix is asymmetric. The adjacency matrix, $\mathbf{A}$ for 
this network is a squared non-symmetric matrix whose elements $A_{i j}$ are defined as

follows:

$A_{i j}=\left\{\begin{array}{cc}l_{i j} & \text { if there is a link from } i \text { to } j \text { having weight } l_{i j} \\ 0 & \text { if } i \text { equals } j\end{array}\right.$

ii) Un-weighted directed networks. This is a simplified representation of the weighteddirected network in which no link weight is quantified but we still consider the direction of the links joining the nodes. There is a link from patch $i$ to $j$ if there is a potential flow of organisms in this direction which exceeds a predefined threshold value (Bodin et al. 2006). This network is represented by a binary directed graph where the weight $l_{i j}$ is 1 if there is a link from node $i$ to node $j$, or 0 otherwise. The elements of the asymmetric adjacency matrix are thus defined as follows

$A_{i j}=\left\{\begin{array}{l}1 \text { if there is a link from } i \text { to } j \\ 0 \text { otherwise }\end{array}\right.$

iii) Un-weighted undirected networks. The simplest representation of a landscape network is the un-weighted undirected graph. In this case we consider two nodes as connected if there is a connection between the corresponding patches irrespectively of its direction. The link weights are 1 or 0 if the corresponding nodes are connected by a link or not, respectively. Thus, the adjacency matrix is a binary symmetric matrix whose elements are defined as follows:

$$
A_{i j}= \begin{cases}1 & \text { if there is a link from } i \text { to } j \text { or from } j \text { to } i \\ 0 & \text { otherwise }\end{cases}
$$

In Table 1 we illustrate the three types of landscape network representations and their respective adjacency matrices.

Insert Table 1 about here 


\section{"Classical" network centrality measures}

Here we present some centrality measures used in studying various different kind of networked systems (Costa et al. 2007, Jordán et al. 2007, Wasserman and Faust 1994).

i) Degree centrality $D C(i)$, is simply the number of links of a node $i$, i.e.,, the number of patches that have a functional connection from or to the patch $i$. In a directed network we will distinguish two types of degree centralities: in-degree and out-degree centralities. The in-degree centrality $D C_{i n}(i)$ is the number of links which terminate in patch $i$ in the landscape. The out-degree centrality $D C_{\text {out }}(i)$ is the number of links that originate from the patch $i$ (Harary 1969). If a landscape has link weights then the in- and out-degree centralities are calculated by summing up the link weights for all the links terminating or originating, respectively, at the corresponding patch.

ii) Betweenness centrality $B C(k)$ is defined as the fraction of shortest paths going through a given node $k$. If $\rho(i, j)$ is the number of shortest paths from node $i$ to node $j$, and $\rho(i, k, j)$ is the number of these shortest paths that pass through node $k$ in the network, then the betweenness centrality of node $k$ is given by (Wasserman and Faust 1994; Freeman 1978):

$$
B C(k)=\sum_{i} \sum_{j} \frac{\rho(i, k, j)}{\rho(i, j)}, \quad i \neq j \neq k
$$

In weighted networks the shortest paths could be defined as the sum of the link weights.

iii) Closeness centrality $C C(i)$ is the sum of the distances from node $i$ to all other nodes in the network, where the distance $d(i, j)$ is defined as the number of links in the shortest path from node $i$ to node $j$. The closeness centrality of node $i$ is given by the following expression (Wasserman and Faust 1994; Freeman 1978): 
$C C(i)=\frac{N-1}{\sum_{j} d(i, j)}$

In weighted networks the distance could be defined as the sum of the link weights. The closeness centrality cannot be calculated for all patches in a disconnected landscape because the distance between un-connected patches is infinite or just undefined.

iv) Eigenvector centrality EC(i) was introduced by Bonacich $(1972,1987)$ and is defined using the principal eigenvector of the adjacency matrix A. EC(i) of node $i$ is defined as the $i$ th component of the eigenvector $\mathbf{e}_{1}$ that corresponds to the largest eigenvalue of A (principal eigenvalue):

$$
E C(i)=e_{1}(i)
$$

The eigenvector centrality has some limitations when applied to the different types of network representations previously presented (Borgatti and Everett 2006). First, it cannot, in its original form, be unambiguously defined for directed networks. Furthermore, it assigns zeros to all patches which are not situated in the largest component (i.e., a subset of nodes where there exist a path between each and every pair of nodes) of the network even if they are highly central in their respective components (Borgatti and Everett 2006).

\section{Subgraph centrality}

One of the authors (EE) has recently introduced a metric characterizing certain aspects of importance of a node in a network which is named the "subgraph centrality" $S C(i)$ (Estrada and Rodríguez-Velázquez 2005). This metric characterizes the participation of a node in all structural motifs (e.g., triangles, squares, etc.) in the network. The participation of a node in a motif is quantified by means of the so-called 
closed-walks (CWs). A walk of length $r$ is a sequence of nodes $v_{1}, v_{2}, \cdots, v_{r}, v_{r+1}$ such that for each $i=1,2 \cdots, r$ there is a path from $v_{i}$ to $v_{i+1}$. A closed walk (CW) is a walk in which $v_{r+1}=v_{1}$ (Harary 1969). A particular case of $\mathrm{CW}$ is the cycle, in which all nodes in $v_{1}, v_{2}, \cdots, v_{r}, v_{r+1}$ are different, but in general a closed walk can involve the same node more than once. In a network representation of a fragmented landscape, these CWs represent different movement pathways within the landscape that terminates at the originating patch.

If we consider a particular node $i$, the total number of CWs of length $r$ originating (and terminating) at $i$ is designated by $\mu_{r}(i)$. The general idea behind the subgraph centrality measure is to relate a node's centrality to the number of CWs of different lengths starting at a given node. However, the sum of CWs of all lengths starting and ending at a given node is infinite, i.e.,, $\sum_{r=0}^{\infty} \mu_{r}(i)=\infty$, which would makes this measure useless. This difficulty is resolved by introducing a weighting scheme that makes that the sum of weighted CWs converge to a definite value (Estrada and Rodríguez-Velázquez 2005).

$$
S C(i)=\sum_{r=0}^{\infty} \frac{\mu_{r}(i)}{r !}=\sum_{r=0}^{\infty} \frac{\left(\mathbf{A}^{r}\right)_{i i}}{r !}
$$

Thus, a CW of length two is weighted by a factor of $1 / 2$, and a CW of length three is weighted by a factor of $1 / 6$ and so on. In general, a $\mathrm{CW}$ of length $r$ is weighted by a factor of $1 / r$ !, which makes the sum of weighted CWs converge, and also fulfill the intuition that the longer closed walks are of less importance in defining a nodes level of centrality (see further explanations in Estrada and Rodríguez-Velázquez 2005). 
For practical reasons we need to truncate the infinite sum given by expression (7).

In doing so we will stop the calculation for the value of $r$ such that

$$
\frac{\mu_{r}}{r !} \leq 10^{-4}
$$

Furthermore, in a binary and undirected representation of a network, (7) converges to the following expression:

$$
S C(i)=\sum_{r=0}^{\infty} \frac{\mu_{r}}{r !}=\sum_{j=1}^{N}\left[\gamma_{j}(i)\right]^{2} e^{\lambda_{j}}
$$

where $\gamma_{j}(i)$ is the $i$ th component of the $j$ th eigenvector of the adjacency matrix $\mathbf{A}$ and $\lambda_{j}$ is the corresponding $j$ th eigenvalue (Estrada and Rodríguez-Velázquez, 2005).

Finally, as seen from an ecological perspective, if a patch in a landscape has a large subgraph centrality, a species would be able to move from that particular patch to a large number of other patches - and then return - by using predominantly closed walks of small lengths.

\section{Centrality measure correlations}

Of interest is to examine to what extent the different centrality measures capture certain distinct aspects of the patches' structural positions, and to what extent the different measures overlap (correlate) in this regard. Furthermore, it is of interest to study to what extent the different centrality measures depend on the different networks representations described earlier. In order to enable such analysis, we calculated the inand out-degree, the betweenness, the in- and out-closeness and the subgraph centrality for all nodes, in all types of network representations, and correlated the resulting values for all centrality measures for all nodes. Hence, we (1) tested the degree of correlation 
between the values for the different centralities for each network representation, and we (2) correlated the centralities among the three different types of network representations.

In addition to the centrality measures mentioned above we also calculated the eigenvector centrality for the simple graph representation of the network, which has a symmetric adjacency matrix allowing such calculations.

Furthermore, to examine to what extent the different centrality measures overlapped we use the principal component (PC) method (Gorsuch 1983) to reduce the sixdimensional centrality space as much as possible while still accounting for most of the variance of the centrality measures. In order to obtain a clear pattern of loadings we will use a typical strategy known as Varimax, which rotates the factors in an orthogonal way (Gorsuch 1983).

\section{Clumpiness coefficient}

A network characteristic that has received fairly little attention is how close to each other the most central nodes in a network are. We believe that such characteristics could, however, be of particular interest in studying some aspects of a landscape's spatial configuration. If for instence, a set of patches, each with high degree centralities, are also directly connected to each other, one might expect a high degree of organism movements confined within such a set.

One measure that tries to capture this network characteristic is the assortativeness introduced by Newman (2002). This measure quantifies whether the most connected nodes in a network are connected to each other or to the least connected ones. Newman proposed measuring the Pearson correlation coefficient of the degree-degree correlation to quantify the assortativeness (Newman 2002). However, this measure only takes into 
account the pairs of connected nodes and does not say anything about the pairs of nodes separated at topological distance larger than one. In order to include these pair of nodes in the analysis we here define a clumpiness coefficient $\Lambda(C)$ for the centrality measure $C$.

The clumpiness coefficient is defined as the averaged value of the product of the standardized centrality for all pairs of nodes $\hat{C}_{i} \hat{C}_{j}$ in the network divided by the square of the corresponding topological distance $d_{i j}$ separating them,

$$
\Lambda(C)=\sum_{i<j}^{N} \frac{\hat{C}_{i} \hat{C}_{j}}{\left(d_{i j}\right)^{2}}
$$

The standardization of the centralities is carried out by $\hat{C}_{i}=\frac{C_{i}-\langle C\rangle}{s}$, where $\langle C\rangle$ is the average and $s$ is the standard deviation of the centrality. This standardization guarantees that we can compare centrality measures which have very different values as they have an average value of zero and standard deviation of one. As can be seen from this expression, when the most central nodes are directly connected, $d_{i j}=1$, the clumpiness reaches its maximum. However, when the most central nodes are far away from each other, $d_{i j}>>1$, the clumpiness is reaching its minimum. Here we are using the topological distance between the pairs of nodes but in other cases the topological distance $d_{i j}$ could be replaced by the effective distance (i.e., the link weights) separating the patches in order to obtain a clumpiness estimate of a centrality measure taking the effective distances into account.

Expression (10) can be obtained from a direct vector-matrix-vector multiplication procedure (Estrada, et al. 1997; Estrada and Rodríguez 1997). Let c be a column vector 
of the standardized centrality measures and $\mathbf{R}$ the matrix of reverse squared distances between pairs of nodes in the graph. Then the clumpiness coefficient is obtained by,

$$
\Lambda(C)=\frac{1}{2}\left(\mathbf{c R c}^{T}\right)
$$

where $T$ stands for the transpose of the vector. Some generalizations and statisticalmechanics interpretation of the clumpiness coefficient have been recently published by Estrada et al. (2008).

\section{Simulation of patch losses}

Ultimately, our interest in the different centrality measures lies in their ability to assess the contribution of a patch to various aspects of the connectivity of a fragmented landscape. In this study we estimated this ability by simulating the removal of patches, ten-by-ten, starting with the most central nodes according to different centrality measures. This strategy has been applied previously to different kinds of complex networks (Estrada 2006; Estrada 2007b). We selected two different network descriptors to assess the centrality measures' abilities to identify patches that characterize the landscape network as a whole. First, we analyzed how the network cliquishness is affected by the removal of the patches. By cliquishness we mean to what extent links tend to be distributed to certain well-connected sets (cliques) of nodes, or if just distributed randomly. High cliquishness would imply high local neighborhood connectivity. In a heavily fragmented landscape, high local connectivity implies access to a fair amount of nearby habitat patches thus providing home ranges of sufficient sizes (cf. Bodin et al. 2006). As a cliquishness measure we selected the Watts-Strogatz clustering coefficient, which is defined as (Watts and Strogatz 1998) 
$C_{i}=\frac{\text { number of triangles connected to node } i}{\text { number of triples centered on node } i}$

The second parameter to be considered is the size of the largest component. If the size of the largest component (in terms of the number of patches it contains) is big in comparison to the total number of patches in the landscape, the level of connectivity can be interpreted as high.

\section{RESULTS}

\section{Weighted directional Madagascar network}

In Fig. 2A we show, for every patch, the intercorrelations between every pair of the centrality measures studied of the weighted directional version of the Madagascar landscape network. The most evident characteristic that can be observed in this plot is the generally low level of correlation between many of the pairs of centrality measures. This clearly shows that different centrality measures are ranking the nodes according to different criteria. The most correlated centralities are the subgraph centrality and the degree centralities, which all show some linear interdependence among each other. On the other extreme we find the closeness centralities, which are not well correlated with neither of the other measures. The differences in the criteria of centrality measures are reflected in the ordering of the patches according to their centrality scores. In Table 2 we give the Spearman correlation coefficients for the different rankings introduced by these centrality measures. These statistics represent the relationship between different rankings on the same set of patches. That is, these coefficients measure the correspondence between two rankings, and assess the significance of this correspondence. If two rankings are the same the Spearman coefficient is equal to 1 and if one ranking is the reverse of the other the coefficient has the value -1 . In general, this coefficient lies between -1 and 1 , 
and increasing absolute values imply increasing agreement between the rankings. As can be seen in Table 2 most of the values of the Spearman correlation coefficient indicate that there is a poor correlation between the different rankings. The best agreements are obtained between the subgraph centrality and the degree centralities. These different rankings beg the question of how to obtain a relevant global ranking of the overall level of centrality of nodes in a landscape network.

Using the principal component (PC) method we have been able to reduce the sixdimensional centrality space to three dimensions where three principal components $\left(P C_{1}\right.$, $P C_{2}$ and $P C_{3}$ ) account for $80 \%$ of the variance of the centrality measures. There are three centrality measures that load in $P C_{1}$ (the two degree centralities and the subgraph centrality). $P C_{2}$ accounts for the betweenness and in-closeness centralities and $P C_{3}$ only accounts for the out-closeness centrality. We have then carried out a Varimax rotation (Gorsuch 1983) to orthogonalize the principal components and obtain their structural interpretation. In Fig. 2B we plot the first two components $\left(P C_{1}\right.$ and $\left.P C_{2}\right)$ after this rotation. The two degree centralities and the subgraph centrality have positive contributions to $P C_{1}$. Since the degree centralities measure the number of functional connections (i.e., links) with the surrounding patches, and subgraph centrality measures the level of participation of a patch in structural motifs like triangles, squares, etc, $P C_{1}$ can be interpreted as a measure of the total dispersal of organisms through the corresponding patch.

On the other hand, the second principal component is dominated by the betweenness centrality and in-degree closeness centrality. Patches with high scores of the betweenness centrality have previously been shown to help bring together otherwise 
largely separated groups of patches (Bodin \& Norberg 2007). If these "bridging" patches, also known as cutnodes, are removed, the connected landscape would risk being separated into significantly smaller compartments (which our experiments of node removals presented further on also confirm). Consequently, the principal component $P_{2}$, can be understood as a measure of the corresponding contribution of a patch in upholding the large-scale connectivity of the landscape. In Table 2 we have included the Spearman rank correlation coefficients for the three principal components. It can be seen that the ranking introduced by $P C_{1}$ agrees very well with the rankings introduced by the degree and subgraph centrality. The highest coefficient is obtained for the subgraph centrality. The ordering introduced by $P C_{2}$ correlates very well with the ranking introduced by the in-closeness centrality and the betweenness centrality and the ordering introduced by $\mathrm{PC}_{3}$ agrees with the one introduced by the out-closeness centrality.

Insert Table 2 and Fig. 2 about here.

\section{Unweighted (binary) directional Madagascar network}

We calculate the centrality measures for all nodes and obtain the inter-centrality correlations, which are illustrated in Fig. 3. Betweenness and closeness centralities are exactly the same as for the weighted version of the network as we did not consider the link-weights in these calculations. As can be seen there is, again, a generally low level of correlation between many of the pairs of centrality measures. However, the correlation between the degree centralities is significantly higher for the binary-directed than for the weighted directed network. 
When analyzing the Spearman rank correlations between the centrality measures we can observe (see Table 3 ) that there is a high level of correlation between the rankings introduced by degree centralities and subgraph centrality. The other two rankings which are in some way correlated are the ones introduced by in-closeness and betweenness centralities.

\section{Insert Fig. 3 about here.}

The factor analysis revealed three principal components accounting for $88 \%$ of the variance of the original centrality measures. As in the previous case the first principal component, $P C_{1}$ is described by the degree and subgraph centralities. The second factor $P C_{2}$ is represented only by the betweenness centrality. Thus, the interpretation of these factors is the same as before. In Table 3 we can see that there are high rank correlations between the $P C_{1}$ and the two degree centralities as well as subgraph centrality. As before, the second principal component is rank correlated to the betweenness but in this case also with the out-closeness centrality instead of the in-closeness centrality.

Insert Table 3 about here.

In Table 4 we show how the centrality scores calculated for the weighted version correlate with the scores calculated for the binary version of the network representation. By analyzing the diagonal entries of the table, it is possible to compare the same centrality measure of the two network representations of the landscape. As can be seen the subgraph and the degree centralities are fairly correlated (correlation coefficient $=0,73$ and 0,66 ), but some information is obviously lost when the link weights are omitted from the analysis (cf. Scotti et al. 2007). For example, only $50 \%$ of the patches in the $P C_{1}$ top ten coincide for both types of networks (data not shown). Betweenness and closeness 
centrality were calculated without taking link weights into account, thus their correlation coefficients are 1.

Insert Table 4 about here.

\section{Binary undirected network}

In Fig. 4 we illustrate the correlation between every pair of centrality measures in this simple graph representation of the Madagascar landscape. As before there is a low level of correlation between many of the pairs of centrality measures. However, the subgraph and degree centralities display some correlations, as do the subgraph and eigenvector centralities. In the latter case the correlation is significantly reduced by the fact that eigenvector centrality assigns zero centrality for all patches which are not within the largest connected component of the network.

\section{Insert Figure 4 about here.}

In Table 5 we give the Spearman correlation coefficient for the different rankings of patches according to the centrality measures analyzed. There is a large coincidence in the rankings introduced by degree, subgraph and eigenvector centrality. Surprisingly, the eigenvector centrality introduces a ranking of patches which is highly correlated to the one introduced by the closeness centrality. The ranking introduced by the betweenness centrality is very unique as it does not correlate with any of the other rankings. A factor analysis identifies a principal component in which degree, eigenvector and subgraph centralities are highly loaded. The second principal component accounts for the betweenness and the third for the closeness. Hence, it is apparent that there are large similarities in the reduced centrality spaces obtained for the three different network representations. The ranking obtained by using $P C_{1}, P C_{2}$ and $P C_{3}$ are also given in Table 5. The first two principal components continue having similar rankings as the 
centrality measures scored in such factors. The third principal component, however, has high Spearman correlation coefficients with most of the centrality measures.

\section{Insert Table 5 about here.}

Table 6 and 7 present the correlations between centralities of patches for the three different network representations of the landscape. The degree of correlation between degree and subgraph centrality is for example slightly reduced when correlating the simple network with the weighted directed one than when correlating the weighteddirected one with the binary directed network. Overall, as before, the level of correlation remains fairly low.

\section{Insert Table 6 and 7 about here.}

\section{How are central patches distributed across the landscape?}

In Fig. 5 we illustrate how the patches with the highest scores of the degree, betweenness, closeness and subgraph centralities for the simple network representation of the Madagascar landscape are distributed. The sizes of the nodes are proportional to the value of the centralities. As can be seen in Figure 5 the most central nodes according to the degree and the betweenness measures are spread across the network. However, the most central nodes according to the subgraph centrality form very compact clusters, which are localized in small regions of the landscape.

If we calculate the clumpiness coefficients for the degree, betweenness, closeness and subgraph centralities of the Madagascar network we confirm quantitatively our observation that subgraph centrality is more clumped than degree centrality in this network, $\Lambda(S C)=634.09$ and $\Lambda(D C)=438.30$. Also, in this landscape the betweenness centrality has a small clumpiness, $\Lambda(B C)=225.09$, which confirms that most of the bridges/cutpoints in the network are spread across the landscape and are not concentrated 
in small regions. By definition the closeness centrality should be the most clumped centrality as it is conceptualized to give higher weight to the nodes which are close to other nodes. In fact, $\Lambda(C C)=732.92$.

\section{Insert Figure 5 about here Vulnerability to patch loss}

Here we simulate how the Madagascar landscape might be affected by the loss of patches. The objective of the simulation is to assess how the loss of the most central patches identified by the different centrality measures affects aspects of the network topology, with consequences on the ecological processes in the landscape. As previously described we selected the most complete description of the Madagascar network for the simulation, which is the weighted directed network. We removed the patches, ten-by-ten, according to their values of the two principal factors identified previously.

The original network is formed by 29 components, which are by definition not connected to each other. In Fig. 6 we present the results of the simulation, where the $x$ axis represents the relative number of patches removed and the $y$-axis represents the relative value of the indicator used. The relative indicators are calculated by dividing the value of the indicator for the disturbed network by that of the original network. By using continuous line we represent the effects produced by the removal of the most central patches according to the $P C_{1}$, i.e.,, the one we propose as representing organism dispersal, while a discontinuous line represents the effects produced by the removal of patches according to factor $\mathrm{PC}_{2}$, i.e., the one we propose as representing the contribution of a patch in upholding the large-scale connectivity. If we consider the network cliquishness (local neighbourhood connectivity) we can see that this parameter is almost 
unaffected when the most central patches ranked by factor $P C_{2}$ are removed. However, the removal of the patches according to the factor $P C_{1}$ produces a dramatic reduction of the cliquishness of the network and by removing about $40 \%$ of patches the cliquishness is reduced by $50 \%$.

The size of the largest component of the network is reduced from 173 patches to only 95 when the 10 most central patches according to factor $P C_{2}$ are removed. The number of patches, however, remains high for the removals based on the $P C_{1}$. The elimination of the top 20 patches according to $P C_{1}$ only reduces the size of the largest component from 173 to 165 patches. However, after $10 \%$ of nodes are removed both factors predict approximately the same effect on the size of the principal component. This somewhat unexpected behavior is mostly a consequence of the severe fragmentation of the principal component following the first removals. Thus, continued patch removals are based on centrality scores which are no longer relevant, therefore it is not surprising that the difference between the two removal schemes is so small.

Insert Fig. 7 about here.

\section{DISCUSSION}

\section{Two families of network centrality}

By comparing the results of the analyses of the different centrality measures in the three different network representations of a fragmented landscape (weighted-directional, unweigthed-directional and unweighted-undirectional) we observed the following generalities described below.

(1) There are (at least) two distinct types (families) of centralities among the set of selected centrality measures for the network studied here. We interpret the first type as potentially relevant in estimating organisms' dispersal at the level of the local 
neighborhood, and the other type as relevant in estimating the ability of individual patches to maintain connectivity beyond the scale of the local neighborhood. The first type includes (in/out) degree-, eigenvector- and subgraph centrality and the second type includes the betweenness centrality for all cases. The simulation of patch removal confirmed the aforementioned differences between the two types of centralities.

(2) These two families of network centrality remain distinct for all three types of network representations. This assessment resulted from the factor analyses of all three network representations where the different centrality measures listed above always ended up in the same principal components, irrespectively of the type of network representation. The closeness centrality, on the other hand, did not show such consistency.

(3) The different types of network representations have quite a significant effect on the assessment of the different centrality scores of individual patches, and that effect seems to be more profound for the family of centrality measures that we interpret as relevant in assessing localized organism dispersal. This assessment is based mainly on the fairly low correlation coefficients of the node centralities in the different network representations. The information contained in the first principal component, which we interpret as related to the dispersal of organisms through a given patch, is significantly affected by the consideration of weight and directionality of links in the landscape network. In comparison, the second principal component $\left(P C_{2}\right)$ is more stable to the differences in network representation. This difference is, however, partly a consequence of not considering the link weights for the calculation of the betweenness centrality. 
(4) Although the different network representations have a profound effect on the ranking of the individual patches' centrality scores, most of the higher scoring patches remain high in centrality irrespectively of the network representation. Thus, even the simple unweighted and undirected network representation is still useful for a coarsegrained assessment of individual patches' importance, but a more detailed assessment of link strength and direction are clearly preferable.

(5) Since the two different families of network centralities remained the same for all different types of network representations, it may suffice to use only one centrality measure from each family for a coarse-grained analysis. Hence, analyses are significantly simplified while still retaining confidence that no important information is lost. According to the rank correlation analysis results (Tables 2, 3 and 5) we recommend using subgraph or degree centrality as a representative of the first principal factor and the betweenness centrality as a representative measure of the second factor.

To summarize, the two families of centrality measures (i.e., the principal components $P C_{1}$ and $P C_{2}$ ) assessed using six different and widely used centrality measures, account for two important but distinct aspects of the landscape connectivity. For instance, the removal of the most central nodes according to $P C_{1}$ will not separate the network into more isolated components but will reduce the local neighborhood connectivity ("cliquishness") of the network, potentially posing limitations on the landscape's ability to attract species with size-requirements on their home range stretching beyond the size of individual patches. On the other hand, $P C_{2}$ accounts for the bridges in the landscape whose removal will separate the landscape into isolated components. Then, the assessment of whether patches are more central (and thus more 
important) than others depends on the aspect of landscape connectivity under consideration. Both aspects of landscape connectivity are important, thus (ideally) patches scoring high in both families of centralities should be conserved while the exploitation of other lower-ranking patches may cause less negative impact on landscape connectivity and its associated ecological processes.

\section{Clumpiness of central patches}

As qualitatively revealed in Fig. 5, and quantified using our proposed clumpiness coefficient, patches with high scores on degree and betweenness centrality are scattered throughout the whole landscape, whereas patches with high scores on subgraph centrality are clumped together in distinct and dense clusters of patches. Hence, although there is a relatively high degree of correlation between subgraph and degree centrality, and despite the fact that they appear in the same principal component in the factor analysis, there are clearly some differences between the two. When it comes to the ranking of the most central patches according to the two measures, there is a high degree of similarity with Spearman rank correlation coefficients larger than 0.9 , irrespective of the network representation (Table 2, 3 and 5). However, as revealed in Fig. 5, the difference appears to reside in the ranking of the patches with low to intermediate scores of centrality. All patches with low to intermediate scores of subgraph centrality are located closely around the highest scoring patches while for degree centrality this characteristic is far less pronounced. Here, some of the patches with relatively high degree centrality stand out on their own, while this never happens for patches with high subgraph centrality. 


\section{Dense clusters of relatively small patches}

In a landscape, in order for a patch to have a high degree centrality, it has to be located geographically close to many other patches. Thus, due to obvious spatial constraints, the mean areas of these patches have to be quite small in comparison with the effective distance the target species can move in the landscape (patches can, by definition, not overlap; thus in order to fit many patches onto a small area they have to be small). Furthermore, a particular node's subgraph centrality score is boosted if its network neighbors have many neighbors themselves, particularly if these neighbors are also neighbors to the originating node (and therefore located within reach from the originating node). This boost is due to the increased number of short-length closed walks resulting from the well connected local network neighborhood. In order to understand more formally the relationship between high subgraph centrality and shorter dispersal distances we exemplify it using an artificial landscape network having $n$ patches in which every patch is connected to all others in the landscape. This kind of networks correspond to the so-called complete graphs, $K_{n}$. We have previously proved mathematically that the largest subgraph centrality in a network having $n$ nodes is obtained for the complete graph $K_{n}$ (Estrada and Rodríguez-Velázquez 2005). That is, among all networks having the same size the maximum subgraph centrality is reached when all nodes are connected to each other. Of course, this network will also display most interpatch movements since an organism situated in one patch can reach any other patch by moving one single step. Similarly, the level of closeness is highest if every node is connected to every other node. Now, let us consider the network $K_{n}-x$ in which the link $x$ is removed from $K_{n}$. Then the number of CWs of length $k$ in $K_{n}-x$ is equal to 
the number of CWs of length $k$ in $K_{n}$ minus the number of CWs of length $k$ in $K_{n}$ containing $x$. Consequently, for all $i, S C(i)$ in $K_{n}-x$ is lower than $S C(i)$ in $K_{n}$. If we consider that the link $x$ in $K_{n}$ is connecting the patches $p$ and $q$, then the distance between $p$ and $q$ in $K_{n}-x$ is increased from 1 to 2 . As a consequence, after the removal of a link in the complete graph the subgraph centrality of any node decreases. The procedure of link removal can be repeated and the previous assertion can be generalized. Thus, in general a high subgraph centrality indicates a network neighborhood that is internally very well connected. In combining this insight with the previous argument on the relationship between patch sizes and number of neighbors, it becomes clear that the distinct groups of patches with high subgraph centrality correspond to areas in the landscape where the habitat has been fragmented into several small, but well connected and closely located, patches.

A reasonable interpretation of such high density clusters is that the patches within the clusters are experienced as being connected by species not being able to move as far in the inhospitable matrix as the target species used to assess the network of fragmented habitat patches in the first place. This is interesting since one drawback using network representations of fragmented landscapes is that the assessed networks are, by definition, specific for the studied target species. If, however, one can use network measures (such as the subgraph and/or the closeness centrality) to identify core areas that would still appear as connected for species not being able to move as far as the target species used to construct the network - a multi-species interpretation of a single species network is possible. 


\section{CONCLUSIONS}

In order to balance between different socio-economic demands on land use, and still provide the generation of essential ecosystem services in a fragmented landscape (Millennium Ecosystem Assessment, 2003; Bodin et al. 2006), there is a need for scientifically reliable methods capable of identifying individual habitat patches that, more than other patches, contribute to upholding important aspects of landscape connectivity. One promising modeling approach that we believe contribute to such development is the graph-theoretical approach used in this study. Here we have specifically studied how different measures of network centrality may help to estimate individual patches contribution to (1) organism movement within the local neighborhood, and (2) the movement of organisms beyond the local neighborhood. We have studied how these measures of centrality depend on the way the network of habitat patches is constructed and thus represented, and we conclude that while the type of network representation have a profound effect of the assessments of different levels of centrality of patches, even the simplest network representation, not taking strength and directionality of organisms flows into account, still provides a coarse-grained assessment of the most important patches according to the two aforementioned aspects of connectivity.

Furthermore we found a significant difference between the reasonably wellcorrelated subgraph and degree centrality measures. Patches with high and intermediate levels of subgraph centrality are clumped together in the landscape whereas patches with high to intermediate levels of degree centrality are more scattered. Thus the clumps of patches with high to intermediate levels of subgrah centrality may be experienced as connected by other species not being able to move as far in the inhospitable matrix as the 
target species used to assess the network of fragmented habitat patches in the first place. This can be seen as a step towards multi-species analyses of networks of fragmented habitat patches.

Although our results are based on analyses of the specific Madagascar landscape network, we believe these finding are more broadly applicable since this particular landscape in Madagascar is not significantly different, as seen from a strictly spatial perspective, from other heavily fragmented landscapes. Naturally, conducting similar

analyses on a range of different landscapes would be preferable and would strengthen our arguments. Furthermore, there are other centrality measures not considered here that might be of relevance in studying landscape connectivity, and there are also reasons to further examine the underlying assumptions behind these and other centrality measures when it comes to the kind of flows they might be appropriate for (Borgatti 2005). Finally, more empirically oriented studies utilizing the graph-theoretical perspective of fragmented landscapes would also be needed.

\section{ACKNOWLEDGEMENTS}

The authors thank Thomas Elmqvist and his co-workers at the Department for Systems Ecology at Stockholm University for providing access to the Madagascar dataset. E.E. thanks the "Ramón y Cajal" program, Spain for partial financial support, and ÖB thanks The Swedish Research Council for Environment, Agricultural Sciences and Spatial Planning (FORMAS) for financial support. The Swedish Research Council and the Department for Research Cooperation at the Swedish International Development Cooperation Agency also provided financial support. The authors thank two anonymous 
referees for suggestions that significantly improved the presentation of this work. 


\section{LITERATURE CITED}

Albert, R. and A.-L. Barabási. 2002. Statistical mechanics of complex networks. Reviews of Modern Physics 74:47-97.

Bascompte, J., and R. V. Solé. 1996. Habitat Fragmentation and Extinction Thresholds in Spatially Explicit Metapopulation Models. Journal of Animal Ecology 65:465473.

Bengtsson, J., P. Angelstam, T. Elmqvist, U. Emanuelsson, C. Folke, M. Ihse, F. Moberg, and M. Nyström. 2003. Reserves, resilience and dynamic landscapes. AMBIO: A Journal of the Human Environment 32:389-396.

Boccaletti, S., V. Latora, Y. Moreno, M. Chavez, and D.-U. Hwang. 2006. Complex networks: Structure and dynamics. Physics Reports 424:75-308.

Bodin, Ö., and J. Norberg. 2007. A network approach for analyzing spatially structured populations in fragmented landscape. Landscape Ecology 22:31-44.

Bodin, Ö., M. Tengö, A. Norman, J. Lundberg, and T. Elmqvist. 2006. The value of small size: loss of forest patches and ecological thresholds in southern Madagascar. Ecological Applications 16:440-451.

Bonacich, P. 1972. Factoring and weighting approach to clique identification. Journal of Mathematical Sociology 2:113-120.

Bonacich, P. 1987. Power and centrality: A family of measures. American Journal of Sociology 92:1170-1182.

Borgatti, S., and M. G. Everett. 2006. A graph-theoretic perspective on centrality. Social Networks 28:466-484.

Borgatti, S. 2005. Centrality and network flow. Social Networks 27: 55-71 
Bunn, A. G., D. L. Urban, and T. H. Keitt. 2000. Landscape connectivity: A conservation application of graph theory. Journal of Environmental Management 59:265-278.

Calabrese, J. M., and W. F. Fagan. 2004. A comparison-shopper's guide to connectivity metrics. Frontiers In Ecology and the Environment 2:529-536.

Campbell Grant, E., W. Lowe, and W. Fagan. 2007. Living in the branches: population dynamics and ecological processes in dendritic networks. Ecology Letters 10:165175.

Costa, L. da F., F. A. Rodrigues, G. Travieso, and P. R. V. Boas. 2007. Characterization of complex networks: a survey of measurements. Advances in Physics, 56:167242.

Estrada, E., L. Rodríguez, and A. Gutiérrez. 1997. Matrix algebraic manipulations of molecular graphs. 1. Graph theoretical invariants based on distances and adjacency matrices. MATCH. Communications in Mathematical and in Computer Chemistry 35:145-156.

Estrada, E., and L. Rodríguez. 1997. Matrix algebraic manipulations of molecular graphs. 2. Harary- and MTI- like molecular descriptors. MATCH. Communications in Mathematical and in Computer Chemistry 35:157-167.

Estrada, E., and J. A. Rodríguez-Velázquez. 2005. Subgraph centrality in complex networks. Physical Review E 71:056103.

Estrada, E. 2006. Network robustness. The interplay of expansibility and degree distribution. European Physical Journal B 52:563-574.

Estrada, E. 2007a. Characterization of topological keystone species. Local, global and "meso-scale" centralities in food webs. Ecological Complexity 4:48-57. 
Estrada, E. 2007b. Food web robustness to biodiversity loss. The roles of connectance, expansibility and degree distribution. Journal of Theoretical Biology 244:296307.

Estrada, E., N. Hatano, and A. Gutiérrez. 2008. "Clumpiness" mixing in complex networks. J. Stat. Mech. P03008.

Fahrig, L. 2003. Effects of habitat fragmentation on biodiversity. Annual Review of Ecology, Evolution, and Systematics 34:487-515.

Freeman, L. C. 1978. Centrality in social networks. Conceptual clarification. Social Networks 1:215-239.

Gorsuch, R. L. 1983. Factor Analysis. Lawrence Erlbaum Associates, Inc. New Jersey.

Gustafson, E. J. 1998. Quantifying landscape spatial pattern: What is the state of the art? Ecosystem 1:143-156.

Hanski, K., and O. Ovaskainen. 2003. Metapopulation theory for fragmented landscapes. Theoretical Population Biology 64:119-127.

Hanski, I. 1994. A practical model of metapopulation dynamics. Journal of Animal Ecology 63:151-162.

Harary, F. 1969. Graph Theory. Addison-Wesley, Reading.

Hargis, C. D., J. A. Bissonette, and J. L. David. 1998. The behavior of landscape metrics commonly used in the study of habitat fragmentation. Landscape Ecology 13:167186.

Jordán, F., Z. Benedek, and J. Podani. 2007. Quantifying positional importance in food webs: A comparison of centrality indices. Ecological Modelling 205:270-275. 
Keitt, T. H., D. L. Urban, and B. T. Milne. 1997. Detecting critical scales in fragmented Llndscapes. Conservation Ecology 1 (online).

Lee, J. T., and S. Thompson. 2005. Targeting sites for habitat creation: an investigation into alternative scenarios. Landscape and Urban Planning 71:17-28.

Li, H., and J. Wu. 2004. Use and misuse of landscape indices. Landscape Ecology 19:389-399.

Lundberg, J., and F. Moberg. 2003. Mobile link organisms and ecosystem functioning: Implications for ecosystem resilience and management. Ecosystems 6:87-98.

Meffe, G. K., L. A. Nielsen, R. L. Knight, and D. A. Schenborn. 2002. Ecosystem management: Adaptive, community-based conservation. Island Press, Washington DC.

Millennium Ecosystem Assessment. 2003. Ecosystems and human well-being. Island Press, Washington DC.

Minor, E. S., and D. L. Urban. 2007. Graph theory as a proxy for spatially explicit population models in conservation planning. Ecological Applications 17:17711782.

Newman, M. E. J. 2002. Assortative mixing in networks. Physical Review Letters 89: 208701

Newman, M. E. J. 2003. The structure and function of complex networks. SIAM Review 45: $167-256$

Pascual-Hortal, L., and S. Saura. 2006. Comparison and development of new graph-based landscape connectivity indices: towards the prioritization of habitat patches and corridors for conservation. Landscape Ecology 21:959-967. 
Scotti, M., J. Podani and F. Jordán. 2007. Weighting, scale dependence and indirect effects in ecological networks: A comparative study. Ecological Complexity 4:148-159.

Strogatz, S. H. 2001. Exploring complex networks. Nature 410:268-275.

Taylor, P. D., L. Fahrig, K. Henein, and G. Merriam. 1993. Connectivity is a vital element of landscape structure. Oikos 68:571-573.

Tischendorf, L. 2001. Can landscape indices predict ecological processes consistently? Landscape Ecology 16:235-254.

Urban, D., and T. Keitt. 2001. Landscape connectivity: A graph-theoretic perspective. Ecology 82:1205-1218.

Verbeylen, G., L. D. Bruyn, F. Adriaensen, and E. Matthysen. 2003. Does matrix resistance influence Red squirrel (Sciurus vulgaris L. 1758) distribution in an urban landscape? Landscape Ecology 18:791-805.

Wasserman, S., and K. Faust. 1994. Social network analysis - Methods and applications. Cambride University Press, Cambridge.

Watts, D. J., and S. H. Strogatz. 1998. Collective dynamics of 'small-world' networks. Nature 393:440-442. 
Table 1. Three different representations of landscape networks using weighted, directed and simple graphs. The definition of the node and link weights and example of the corresponding adjacency matrices are also illustrated.

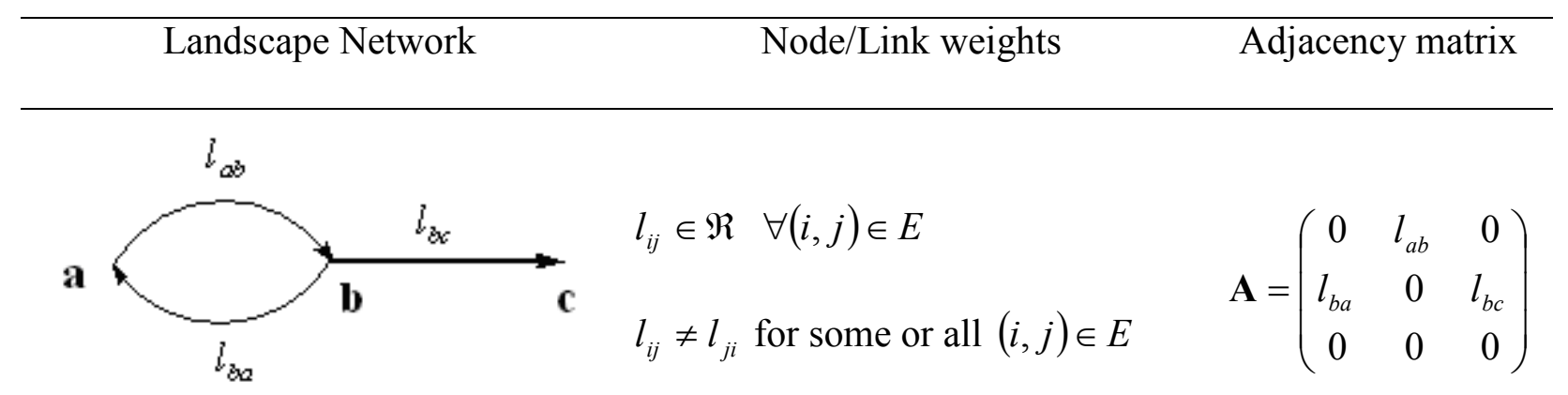

Weighted-Directed

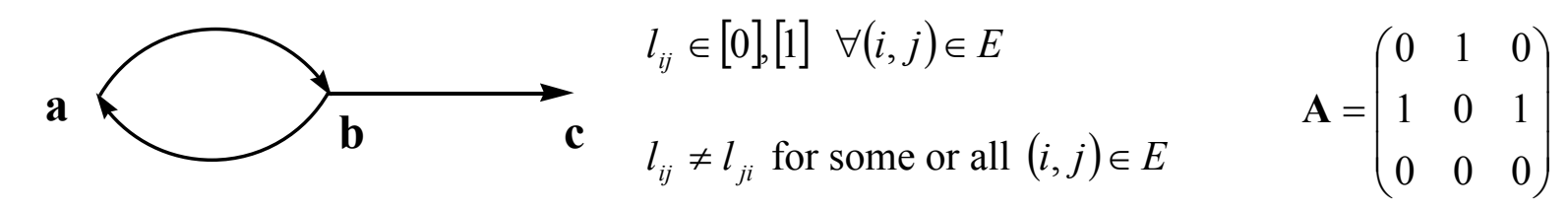

Binary-Directed

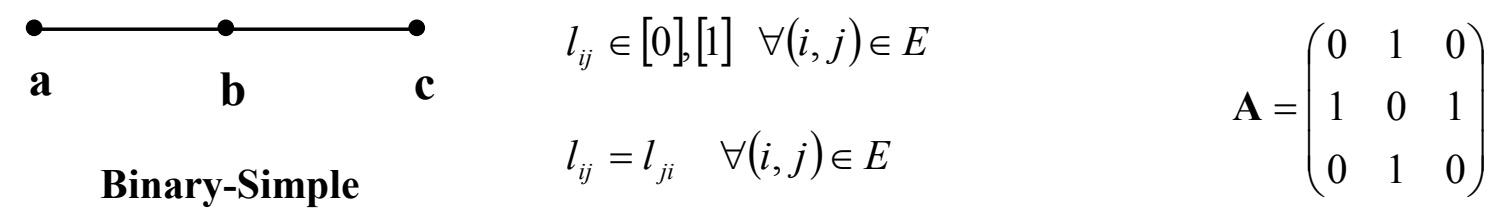


Table 2. Spearman rank correlation coefficients for the different rankings introduced by the centrality measures studied as well as by the two principal components obtained by using the factor analysis for the weighted-directed representation of the Madagascar landscape network. Marked correlations are significant at $\mathrm{p}<0.05$.

\begin{tabular}{|c|c|c|c|c|c|c|c|c|}
\hline & $D C_{o u t}$ & $S C$ & $B C$ & $C C_{i n}$ & $C C_{\text {out }}$ & $P C_{1}$ & $P C_{2}$ & $P C_{3}$ \\
\hline$D C_{i n}$ & 0.810 & 0.908 & 0.287 & 0.374 & 0.316 & 0.866 & 0.206 & 0.027 \\
\hline$D C_{\text {out }}$ & & 0.953 & 0.193 & 0.403 & 0.359 & 0.917 & 0.113 & 0.033 \\
\hline$S C$ & & & 0.205 & 0.395 & 0.350 & 0.938 & 0.141 & 0.022 \\
\hline$B C$ & & & & 0.620 & 0.548 & 0.065 & 0.740 & 0.437 \\
\hline$C C_{\text {in }}$ & & & & & 0.312 & 0.376 & 0.885 & -0.104 \\
\hline$C C_{\text {out }}$ & & & & & & 0.143 & 0.322 & 0.820 \\
\hline$P C_{1}$ & & & & & & & 0.083 & -0.205 \\
\hline$P C_{2}$ & & & & & & & & 0.022 \\
\hline
\end{tabular}


Table 3. Spearman rank correlation coefficients for the different rankings introduced by the centrality measures studied as well as by the two principal components obtained by using the factor analysis for the binary-directed version of the Madagascar landscape network. Marked correlations are significant at $\mathrm{p}<0.05$.

\begin{tabular}{|c|c|c|c|c|c|c|c|c|}
\hline & $D C_{\text {out }}$ & $S C$ & $B C$ & $C C_{i n}$ & $C C_{\text {out }}$ & $P C_{1}$ & $P C_{2}$ & $P C_{3}$ \\
\hline$D C_{i n}$ & 0.935 & 0.937 & 0.509 & 0.517 & 0.521 & 0.943 & 0.134 & 0.278 \\
\hline$D C_{\text {out }}$ & & 0.963 & 0.481 & 0.509 & 0.572 & 0.963 & 0.122 & 0.239 \\
\hline$S C$ & & & 0.408 & 0.508 & 0.574 & 0.966 & 0.111 & 0.201 \\
\hline$B C$ & & & & 0.618 & 0.548 & 0.468 & 0.688 & 0.199 \\
\hline$C C_{i n}$ & & & & & 0.307 & 0.502 & 0.336 & 0.662 \\
\hline$C C_{\text {out }}$ & & & & & & 0.676 & 0.708 & -0.382 \\
\hline$P C_{1}$ & & & & & & & 0.214 & 0.132 \\
\hline$P C_{2}$ & & & & & & & & -0.299 \\
\hline
\end{tabular}


Table 4. Correlation coefficients for the linear regression between centrality measures obtained for two different representations of the same landscape network, i.e.,, weighted network and binary-directed network. Correlation coefficients larger than 0.5 are in bold.

\begin{tabular}{ccccccc}
\hline & \multicolumn{5}{c}{ Weighted Network } \\
\hline Binary Directed & $D C_{\text {in }}$ & $D C_{\text {out }}$ & $S C$ & $B C$ & $C C_{\text {in }}$ & $C C_{\text {out }}$ \\
\hline$D C_{\text {in }}$ & $\mathbf{0 . 6 6}$ & $\mathbf{0 . 6 1}$ & $\mathbf{0 . 5 5}$ & 0.10 & 0.21 & $\mathbf{0 . 5 3}$ \\
$D C_{\text {out }}$ & $\mathbf{0 . 5 5}$ & $\mathbf{0 . 6 6}$ & $\mathbf{0 . 5 4}$ & 0.01 & 0.20 & $\mathbf{0 . 5 7}$ \\
$S C$ & $\mathbf{0 . 5 3}$ & $\mathbf{0 . 6 2}$ & $\mathbf{0 . 7 3}$ & -0.06 & -0.02 & $\mathbf{0 . 5 2}$ \\
$B C$ & 0.13 & -0.10 & 0.00 & $\mathbf{1 . 0 0}$ & 0.40 & 0.11 \\
$C C_{\text {in }}$ & 0.13 & 0.18 & 0.05 & 0.40 & 1.00 & -0.06 \\
$C C_{\text {out }}$ & 0.21 & 0.32 & 0.26 & 0.11 & -0.06 & 1.00 \\
\hline
\end{tabular}


Table 5. Spearman rank correlation coefficients for the different rankings introduced by the centrality measures studied as well as by the two principal components obtained by using the factor analysis for the simple (unweighted-undirected) representation of the Madagascar landscape network. Marked correlations are significant at $\mathrm{p}<0.05$.

\begin{tabular}{lccccccc}
\hline & $S C$ & $B C$ & $C C$ & $E C$ & $P C_{1}$ & $P C_{2}$ & $P C_{3}$ \\
\hline$D C$ & $\mathbf{0 . 9 6 2}$ & 0.485 & $\mathbf{0 . 5 3 3}$ & $\mathbf{0 . 6 4 6}$ & $\mathbf{0 . 7 5 0}$ & -0.163 & $\mathbf{0 . 5 3 2}$ \\
$S C$ & & 0.377 & $\mathbf{0 . 5 5 3}$ & $\mathbf{0 . 7 0 8}$ & $\mathbf{0 . 7 3 5}$ & -0.256 & $\mathbf{0 . 5 4 4}$ \\
$B C$ & & & $\mathbf{0 . 6 6 0}$ & 0.516 & 0.154 & $\mathbf{0 . 5 0 4}$ & 0.338 \\
$C C$ & & & & $\mathbf{0 . 8 6 1}$ & 0.120 & 0.133 & $\mathbf{0 . 5 4 1}$ \\
$E C$ & & & & & 0.360 & 0.005 & $\mathbf{0 . 5 0 9}$ \\
$P C_{1}$ & & & & & & 0.017 & -0.040 \\
$P C_{2}$ & & & & & & & $\mathbf{- 0 . 5 5 2}$ \\
\hline
\end{tabular}


Table 6. Correlation coefficients for the linear regression between centrality measures obtained for two different representations of the same landscape network, i.e.,, weighted network and simple network. Correlation coefficients larger than 0.5 are in bold.

\begin{tabular}{ccccccc}
\hline \multicolumn{7}{c}{ Weighted Network } \\
\hline Simple Network & $D C_{\text {in }}$ & $D C_{\text {out }}$ & $S C$ & $B C$ & $C C_{\text {in }}$ & $C C_{\text {out }}$ \\
\hline$D C$ & $\mathbf{0 . 6 3}$ & $\mathbf{0 . 6 1}$ & $\mathbf{0 . 5 3}$ & 0.08 & 0.20 & $\mathbf{0 . 5 6}$ \\
$S C$ & $\mathbf{0 . 5 5}$ & $\mathbf{0 . 6 0}$ & $\mathbf{0 . 6 8}$ & -0.06 & -0.02 & $\mathbf{0 . 5 5}$ \\
$B C$ & 0.12 & -0.11 & -0.01 & $\mathbf{0 . 9 8}$ & 0.35 & 0.11 \\
$C C$ & 0.22 & 0.32 & 0.20 & 0.32 & $\mathbf{0 . 5 7}$ & $\mathbf{0 . 7 0}$ \\
$E C$ & 0.39 & 0.47 & $\mathbf{0 . 5 6}$ & 0.02 & -0.04 & 0.50 \\
\hline
\end{tabular}


Table 7. Correlation coefficients for the linear regression between centrality measures obtained for two different representations of the same landscape network, i.e.,, binarydirected network and simple network. Correlation coefficients larger than 0.5 are in bold.

\begin{tabular}{ccccccc}
\hline & \multicolumn{7}{c}{ Binary Directed Network } \\
\hline Simple Network & $D C_{\text {in }}$ & $D C_{\text {out }}$ & $S C$ & $B C$ & $C C_{\text {in }}$ & $C C_{\text {out }}$ \\
\hline$D C$ & $\mathbf{0 . 9 8}$ & $\mathbf{0 . 9 6}$ & $\mathbf{0 . 7 7}$ & 0.08 & 0.20 & $\mathbf{0 . 5 6}$ \\
$S C$ & $\mathbf{0 . 8 0}$ & $\mathbf{0 . 8 4}$ & $\mathbf{0 . 9 8}$ & -0.06 & -0.02 & $\mathbf{0 . 5 5}$ \\
$B C$ & 0.08 & -0.02 & -0.09 & $\mathbf{0 . 9 8}$ & 0.35 & 0.11 \\
$C C$ & 0.47 & 0.50 & 0.33 & 0.32 & $\mathbf{0 . 5 7}$ & $\mathbf{0 . 7 1}$ \\
$E C$ & $\mathbf{0 . 6 0}$ & $\mathbf{0 . 6 2}$ & $\mathbf{0 . 8 3}$ & 0.02 & -0.04 & 0.50 \\
\hline
\end{tabular}




\section{Fig. captions}

Fig. 1. Southern Androy, southern Madagascar (Landsat image May 2000). The extent of the Landsat image is Lat $25^{\circ} 8^{\prime}$ to Lat $25^{\circ} 23^{\prime} \mathrm{S}$ and Long $45^{\circ} 47^{\prime}$ to $46^{\circ} 12^{\prime}$ E. The black area in the southeast corner is the Indian Ocean, and the filled square in the northeast is the town Ambovombe. The forest patches are identifiable by the distinct dark spots, situated within a matrix consisting of cultivated land (light gray). Patches range in size from $<1-95$ ha and are fairly evenly distributed in the landscape. In the western and northern part of the studied area, the shaded/darker gray zones indicate larger areas classified as potential source areas. Forest habitats constitute approximately $3.5 \%$ of the study area (shaded/darker gray zones are not included). Source: (Bodin et al. 2006).

Fig. 2. (a) Scatterplots for the centrality-centrality correlations in the weightedasymmetric representation of the Madagascar landscape network. The diagonal entries correspond to the distribution of the corresponding centrality measure. (b) Plot of the different centrality measures studied in the space of the two principal components found by using the factor analysis. Observe the clustering of the degree and subgraph centralities in one cluster as well that of in-closeness and betweenness in another cluster.

Fig. 3. Scatterplots for the centrality-centrality correlations in the binary-directed representation of the Madagascar landscape network. The diagonal entries correspond to the distribution of the corresponding centrality measure. 
Fig. 4. Scatterplots for the centrality-centrality correlations in the simple representation of the Madagascar landscape network. The diagonal entries correspond to the distribution of the corresponding centrality measure.

Fig. 5. Modeled landscape network of southern Madagascar showing the distribution of high-centrality patches (unweighted and undirected). The size of each patch (i.e., node) is proportional to its score on (a) Degree centrality, (b) Subgraph centrality, (c) Closeness centrality, and (d) Betweenness centrality.

Fig. 6. Resilience of the Madagascar landscape network to the removal of the most central patches. The ranking of patches is carried out by means of the two principal components (Factor 1 and Factor 2). The resilience is analyzed by considering two different network parameters: clustering coefficient and the size of the largest component. 
Fig. 1

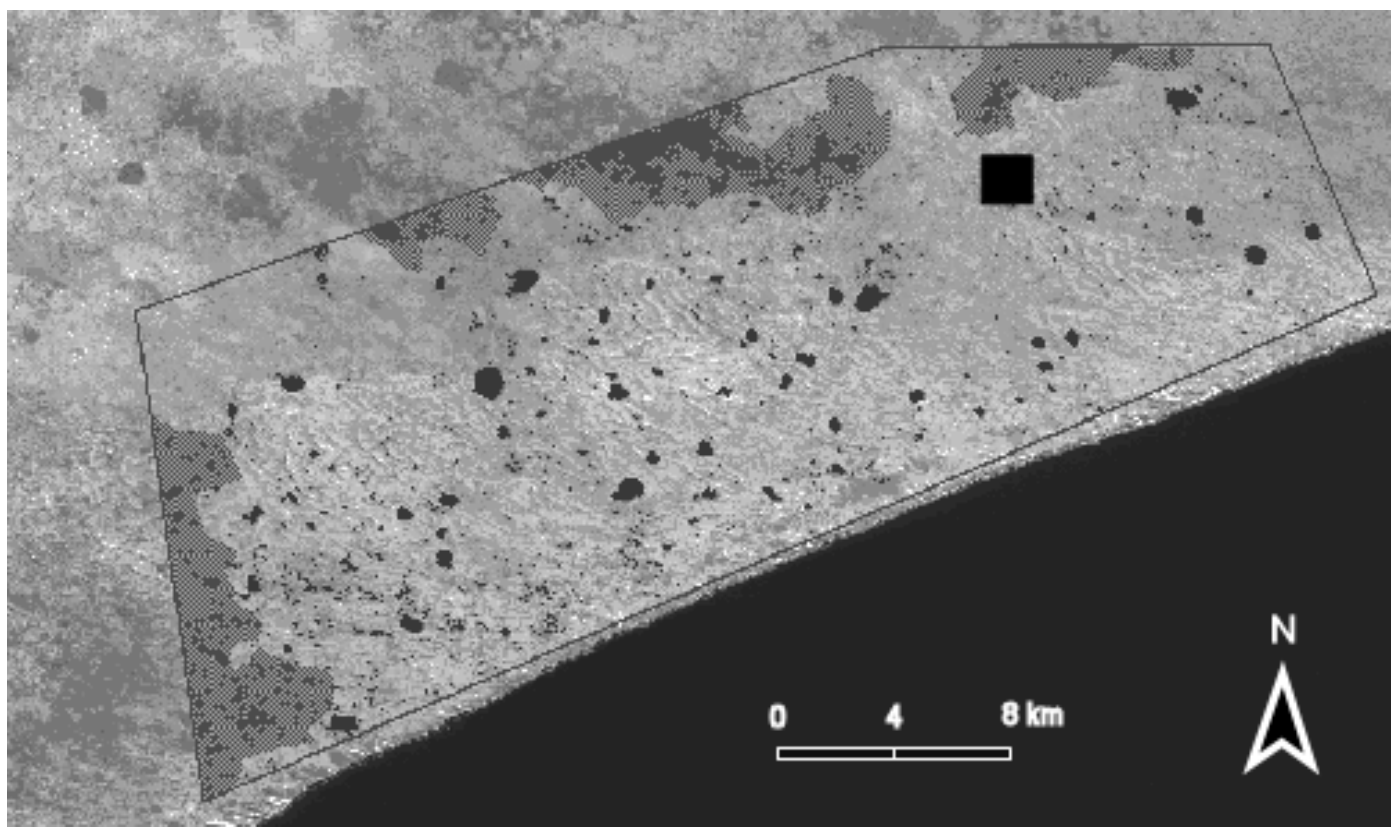


Fig. 2

a)
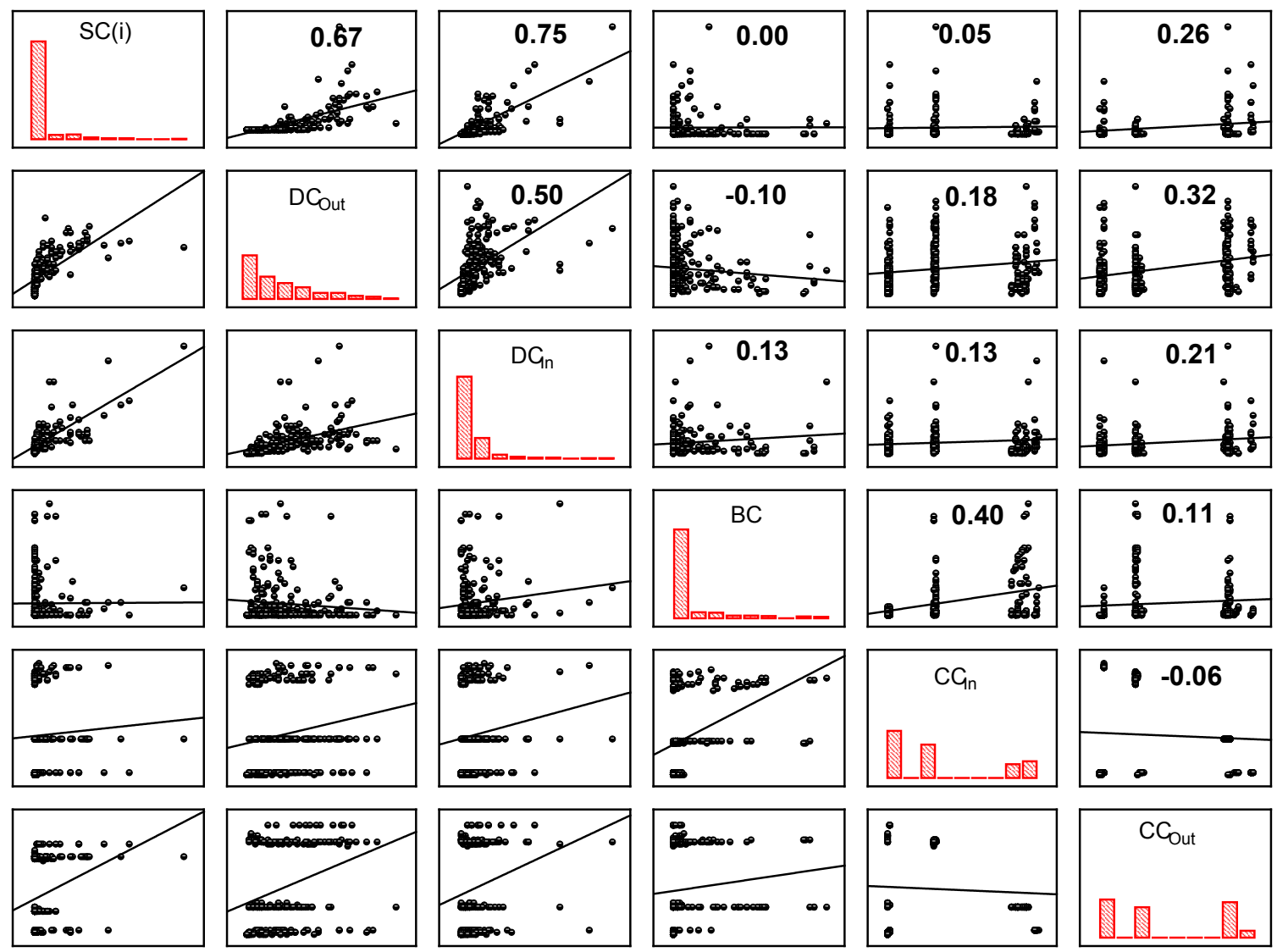

b) 


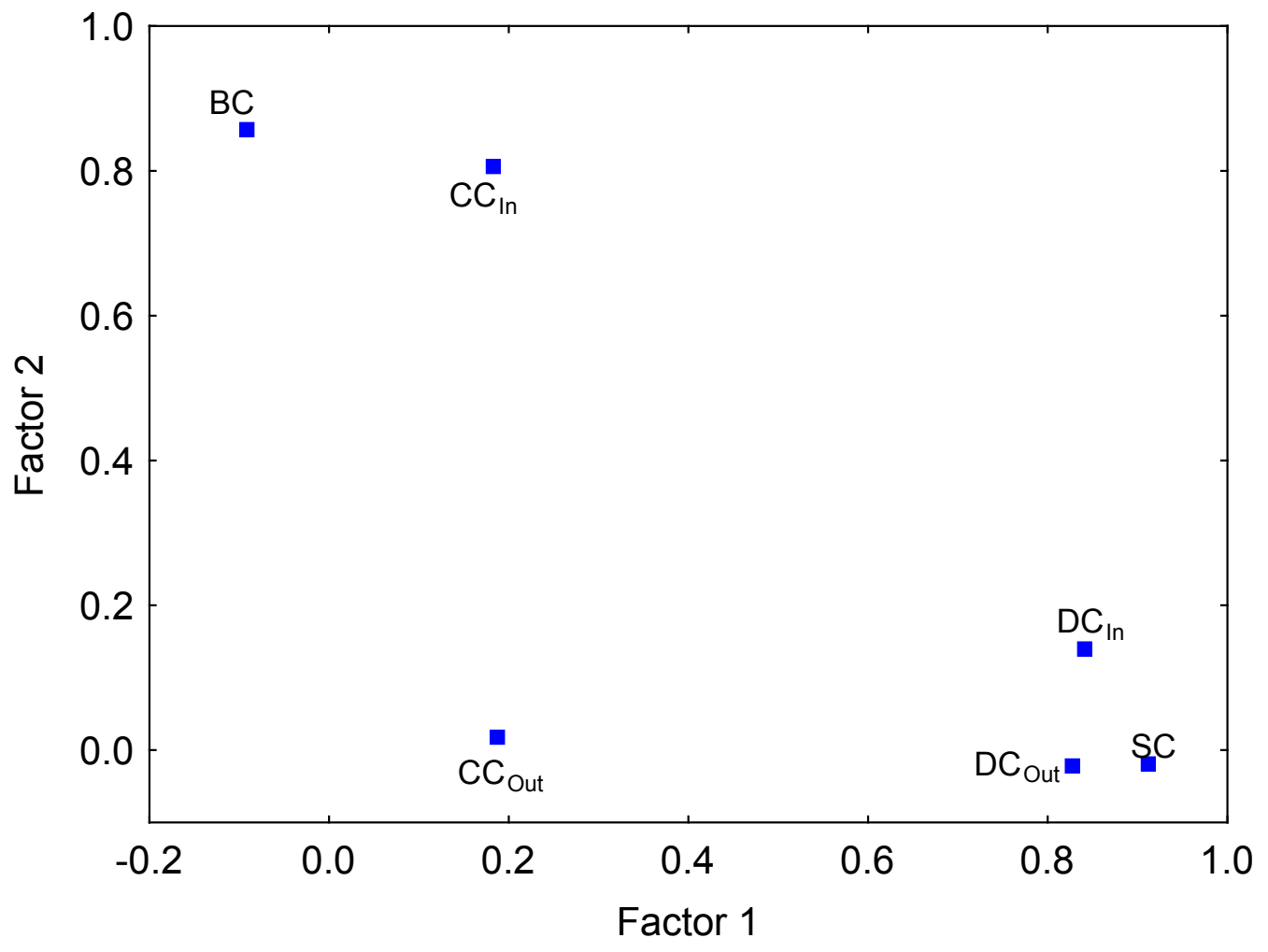

Fig. 3 

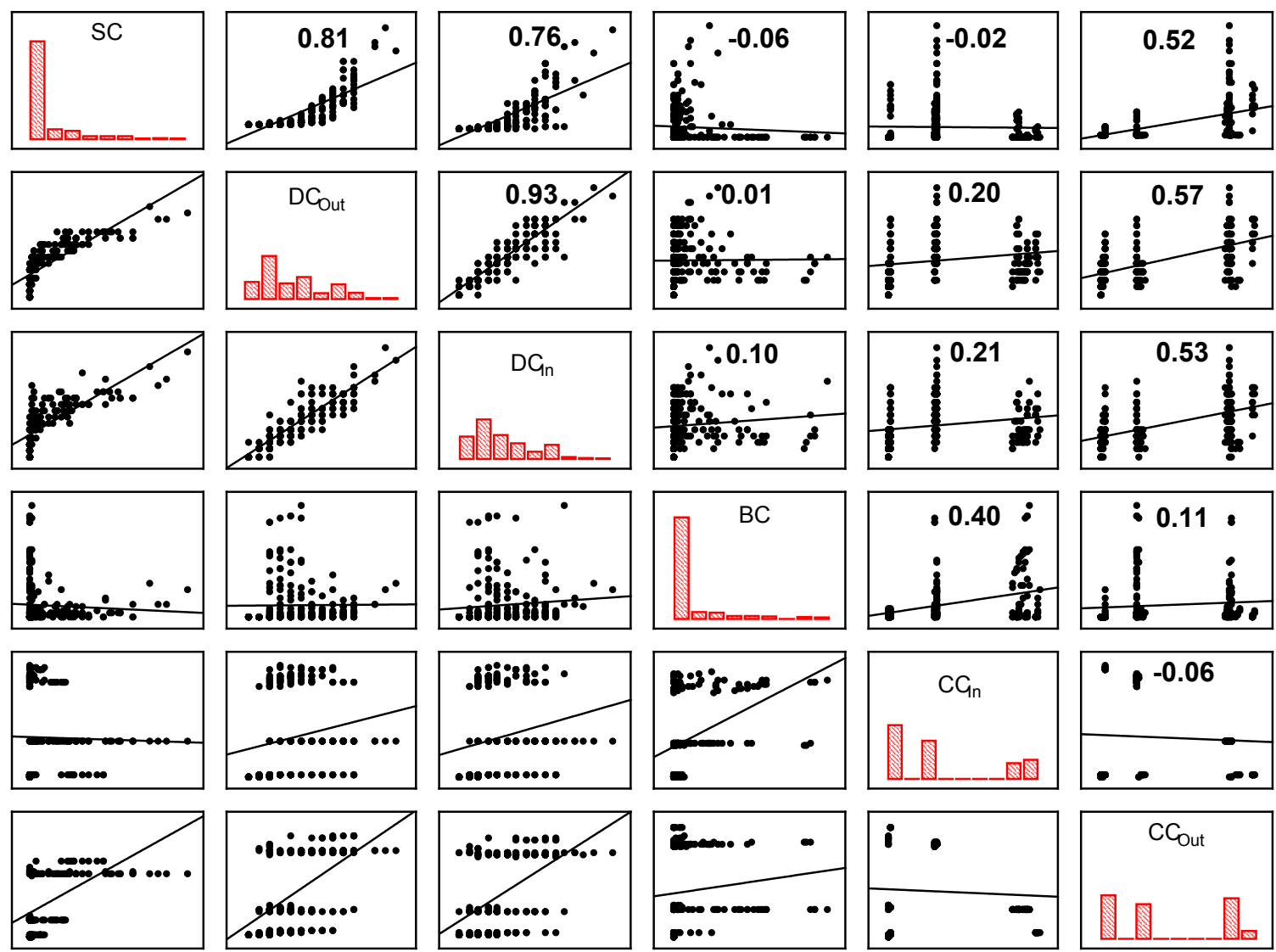
Fig. 4
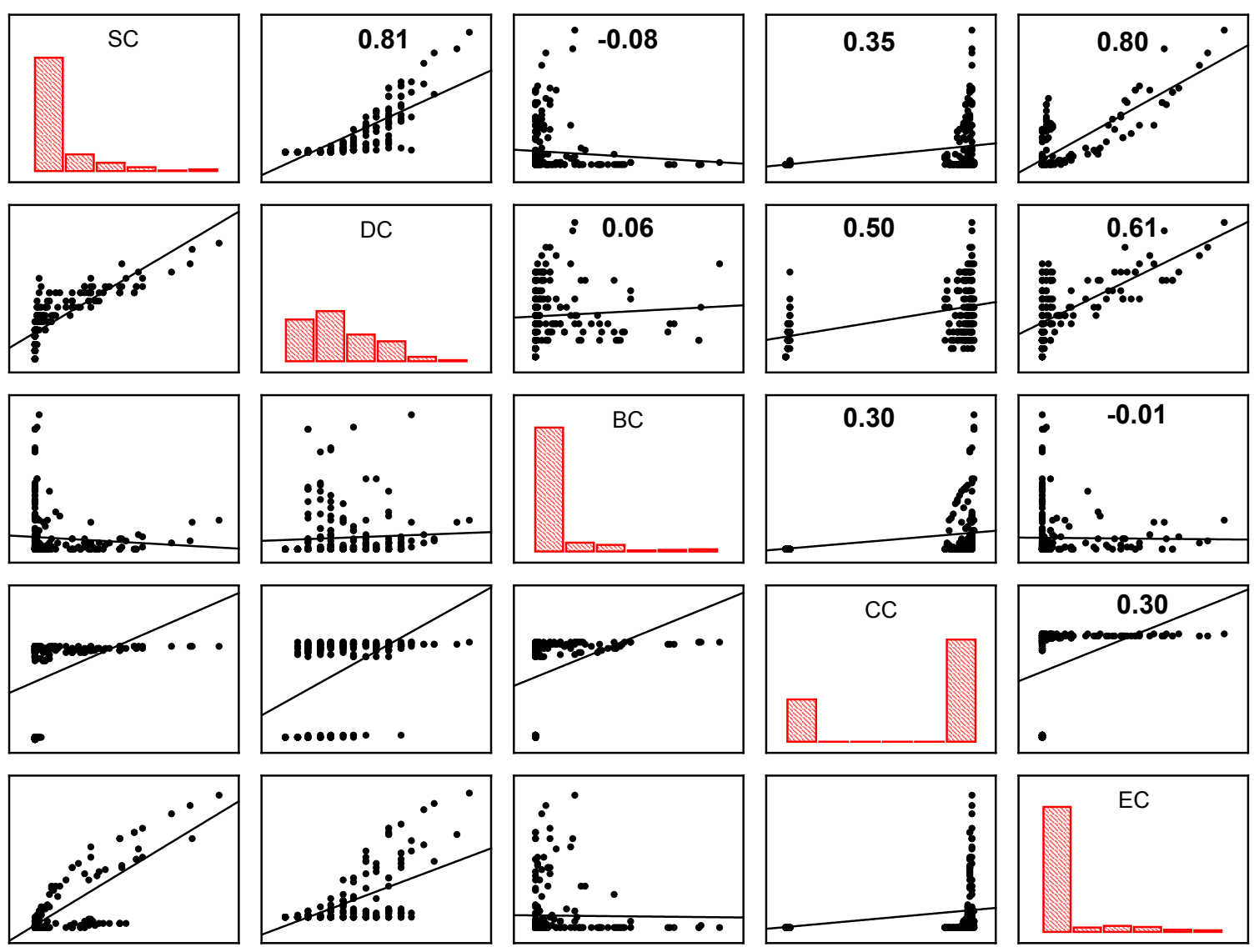

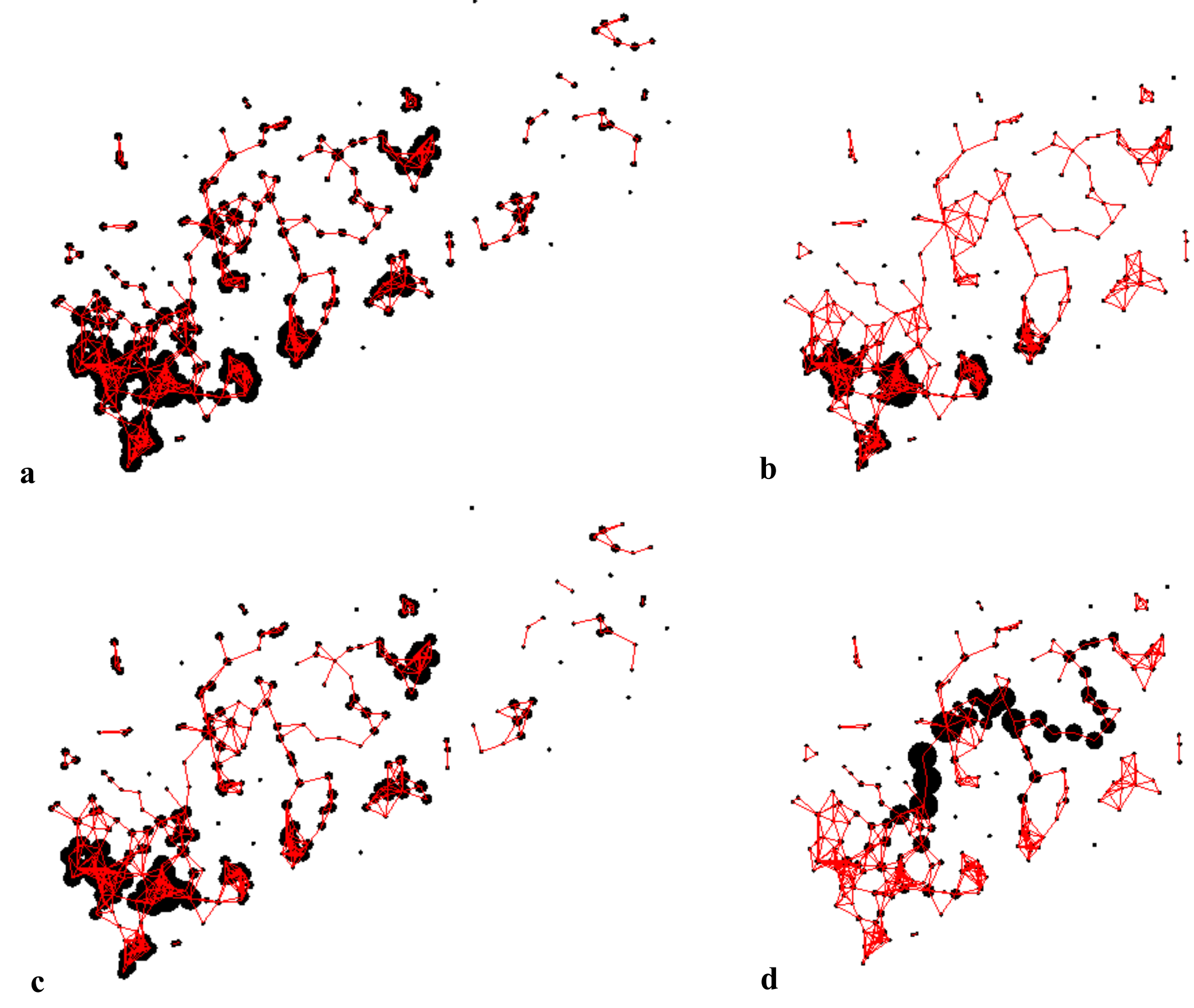
Fig. 6

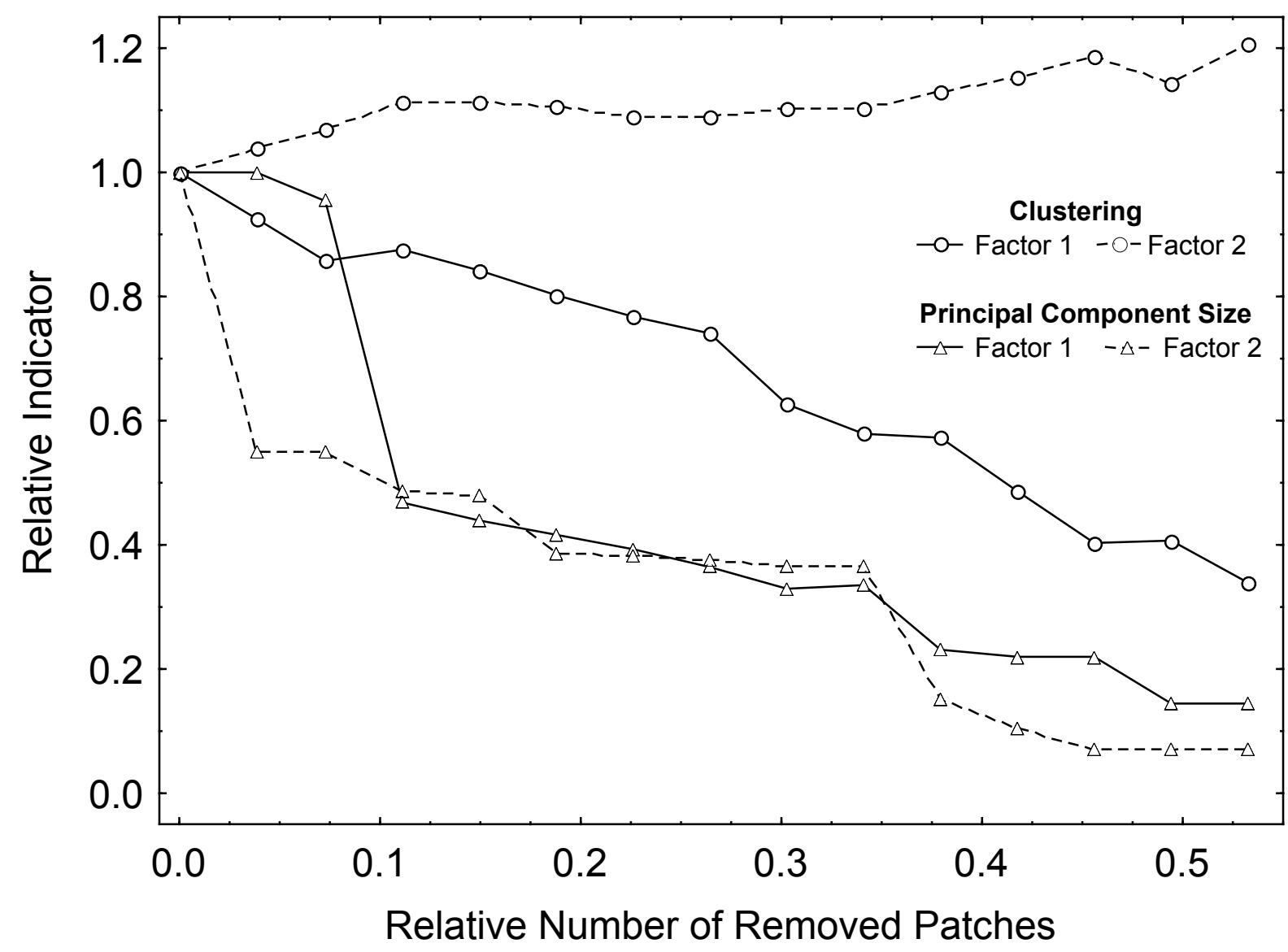

